\title{
Dual-Energy Computed Tomography Compared to Lung Perfusion Scintigraphy to Assess Pulmonary Perfusion in Patients Screened for Endoscopic Lung Volume Reduction
}

\author{
Hester A. Gietema ${ }^{a}$ Kim H.M. Walraven ${ }^{b}$ Rein Posthumab, c, d Cristina Mitea ${ }^{a}$ \\ Dirk-Jan Slebos $^{\mathrm{e}}$ Lowie E.G.W. Vanfleteren ${ }^{f}$ \\ aDepartment of Radiology and Nuclear Medicine, GROWSchool for Oncology and Developmental Biology, \\ Maastricht University Medical Center+, Maastricht, The Netherlands; ${ }^{b}$ Department of Pulmonology, NUTRIMSchool \\ of Nutrition and Translational Research in Metabolism, Maastricht University Medical Center+, Maastricht, \\ The Netherlands; 'Department of Pulmonology, Maastricht University Medical Center+, Maastricht, \\ The Netherlands; ${ }^{d} \mathrm{CIRO}+$, Center of Expertise for Chronic Organ Failure, Horn, The Netherlands; ${ }^{\text {Department }}$ of \\ Pulmonary Diseases, University of Groningen, University Medical Center Groningen, Groningen, The Netherlands; \\ fCOPD Center, Sahlgrenska University Hospital and Institute of Medicine, University of Gothenburg, \\ Göteborg, Sweden
}

\begin{abstract}
Keywords
Bronchoscopic lung volume reduction · Computed tomography, lung · Dual-energy computed tomography · Emphysema $\cdot$ Perfusion scan · Scintigraphy
\end{abstract}

\begin{abstract}
Background: Endoscopic lung volume reduction (ELVR) using one-way endobronchial valves is a technique to reduce hyperinflation in patients with severe emphysema by inducing collapse of a severely destroyed pulmonary lobe. Patient selection is mainly based on evaluation of emphysema severity on high-resolution computed tomography and evaluation of lung perfusion with perfusion scintigraphy. Dual-energy contrast-enhanced CT scans may be useful for perfusion assessment in emphysema but has not been compared against perfusion scintigraphy. Aims: The aim of the study was to compare perfusion distribution assessed with dual-energy contrast-enhanced computed tomography and perfusion scintigraphy. Material and Methods: Forty consecutive patients with severe emphysema, who were screened for ELVR,
\end{abstract}

were included. Perfusion was assessed with $99 \mathrm{mTc}$ perfusion scintigraphy and using the iodine map calculated from the dual-energy contrast-enhanced CT scans. Perfusion distribution was calculated as usually for the upper, middle, and lower thirds of both lungs with the planar technique and the iodine overlay. Results: Perfusion distribution between the right and left lung showed good correlation $(r=0.8)$. The limits of agreement of the mean absolute difference in percentage perfusion per region of interest were $0.75-5.6 \%$. The upper lobes showed more severe perfusion reduction than the lower lobes. Mean difference in measured pulmonary perfusion ranged from $-2.8 \%$ to $2.3 \%$. Lower limit of agreement ranged from $-8.9 \%$ to $4.6 \%$ and upper limit was $3.3-10.0 \%$. Conclusion: Quantification of perfusion distribution using planar $99 \mathrm{mTc}$ perfusion scintigraphy and iodine overlays calculated from dual-energy contrast-enhanced CTs correlates well with acceptable variability.

(C) 2021 The Author(s)

Published by S. Karger AG, Basel

H.A. Gietema and K.H.M. Walraven contributed equally. (c) 2021 The Author(s)

Published by S. Karger AG, Basel

This is an Open Access article licensed under the Creative Commons Attribution-NonCommercial-4.0 International License (CC BY-NC) (http://www.karger.com/Services/OpenAccessLicense), applicable to the online version of the article only. Usage and distribution for commercial purposes requires written permission.
Correspondence to:

Hester A. Gietema, hester.gietema@mumc.nl 


\section{Introduction}

Emphysema is a progressive and debilitating disease characterized by irreversible destruction of lung parenchyma. Emphysema is hallmarked by loss of elastic recoil and air trapping. This air trapping results in hyperinflation, which limits gas exchange and results in chronic dyspnea, reduced exercise tolerance, and poor health-related quality of life. In those patients in whom the parenchymal destruction has advanced to severe emphysema, morbidity and mortality is high. Reducing hyperinflation can result in less dyspnea and better quality of life [1]. The introduction of newer endoscopic noninvasive techniques led to a successful revival of lung volume reduction in the management of patients with chronic obstructive pulmonary disease (COPD) and emphysema. It took a decade of learning in which it became clear that treatment success was highly dependent on patient selection and more specifically on the selection of a suitable target lobe on radiologic imaging [2-4].

Therefore, subjects who meet the clinical and pulmonary function criteria for endoscopic lung volume reduction (ELVR) undergo a high-resolution CT (HRCT) to assess presence, extent, and heterogeneity of emphysema, fissure integrity, and presence of adhesions to define a suitable target lobe. The presence of suspicious pulmonary nodules and/or signs of active infection, bronchiectasis and small airways disease can be a possible contraindication for ELVR $[5,6]$. However, data suggest that even in patients with more homogeneous emphysema, that is, more equal emphysematous destruction in upper and lower lobes, endobronchial valve treatment can also be successful [4]. The role of distribution of perfusion as a measure to identify heterogeneity in functionality in more homogeneous destructed lungs became more important for appropriate target lobe selection.

Consequently, for optimal treatment target selection, the ELVR expert panel advices to perform a perfusion scan for subjects who have relatively homogeneous emphysema [7]. In the most recent published update of the expert panel recommendations, it is suggested to perform a $99 \mathrm{mTc}$ perfusion scintigraphy in all patients screened for ELVR [8]. However, pulmonary perfusion may also be assessed with newer CT techniques, which can be combined with the HRCT in the same session. This could reduce time and costs, but could importantly reduce the diagnostic burden for these highly symptomatic patients.

In contrast to most body materials, iodine has a higher $\mathrm{X}$-ray attenuation at lower photon energies. The introduction of the dual-energy multislice computed tomog- raphy offered the opportunity to create an iodine map by simultaneous scanning at 2 different energies. This iodine map can be used a surrogate marker of pulmonary perfusion. So far, this technique is mainly applied in detection of pulmonary emboli [9] and studies comparing both techniques are limited and results are both techniques with $99 \mathrm{mTc}$ perfusion scintigraphy are variable $[10,11]$. Only limited data on perfusion quantification with both techniques are available in COPD patients. Pansini et al. [12] showed in smokers with severe emphysema, a lower mean attenuation than nonsmokers and smokers without significant emphysema, but the number of included smokers with severe emphysema in this study was limited. In this study, we hypothesized that pulmonary perfusion assessed with an iodine perfusion map acquired with dual-energy contrast-enhanced computed tomography is comparable to perfusion quantification with $99 \mathrm{mTc}$ perfusion in patients with severe emphysema in the diagnostic work-up for ELVR.

\section{Material and Methods}

For this study, we included severe to very severe COPD patients that were screened for ELVR in our hospital between February 2018 and April 2019. Patients were selected for screening on the basis of the presence of severe COPD (FEV1/FVC $<70 \%$, FEV1 $<45 \%$ [post bronchodilator]) and severe hyperinflation: total lung capacity $>100 \%$, residual volume $(\mathrm{RV})>175 \%$, RV/total lung capacity ratio $>0.55$. Patients with relevant comorbidities such asthma phenotype, severe obesity (BMI $>30)$, history of recurrent pulmonary infections, bronchiectasis, immunodeficiency, and/or history of lung surgery were excluded from ELVR. A waiver was received from the Medical Ethical Review Board from Maastricht University Medical Center under METC 2018-0868.

Perfusion scintigraphy was performed directly after intravenous administration of $74 \mathrm{MBq}$ technetium-99m labeled macroaggregated albumin (99mTc-MAA). The patient was examined in supine position and during tidal inspiration, perfusion images were acquired using a low-energy, high-resolution collimator. Four standard projections were obtained: anterior, posterior, right posterior oblique, and left posterior oblique. An energy window of $15-20 \%$ centered at $140 \mathrm{keV}$ and a matrix of $256 \times 256$ pixels were applied and approximately 200,000 counts accumulated per view. For quantification, each lung was divided into 3 equal rectangular regions, in anterior and posterior views. The activity in each lung and in these 6 regions of interest was reported as percentage of total activity (Fig. 1a).

CT scans were performed at a Siemens Somatom Force dualenergy CT scanner $(n=33)$ or a Siemens Somatom Definition Flash CT scanner $(n=7)$ (both Siemens Healthcare, Forchheim, Germany). HRCT included an inspiratory and an expiratory CT. The inspiratory scan was performed at $120 \mathrm{kV}$ and $40 \mathrm{mAs}$, reconstructed at $1-\mathrm{mm}$ axial slices with both a soft (Br40) and edge-enhancing (Br69) reconstruction filter and at coronal and sagittal 3 $\mathrm{mm}$ slices with a soft filter. The expiratory CT was performed at 

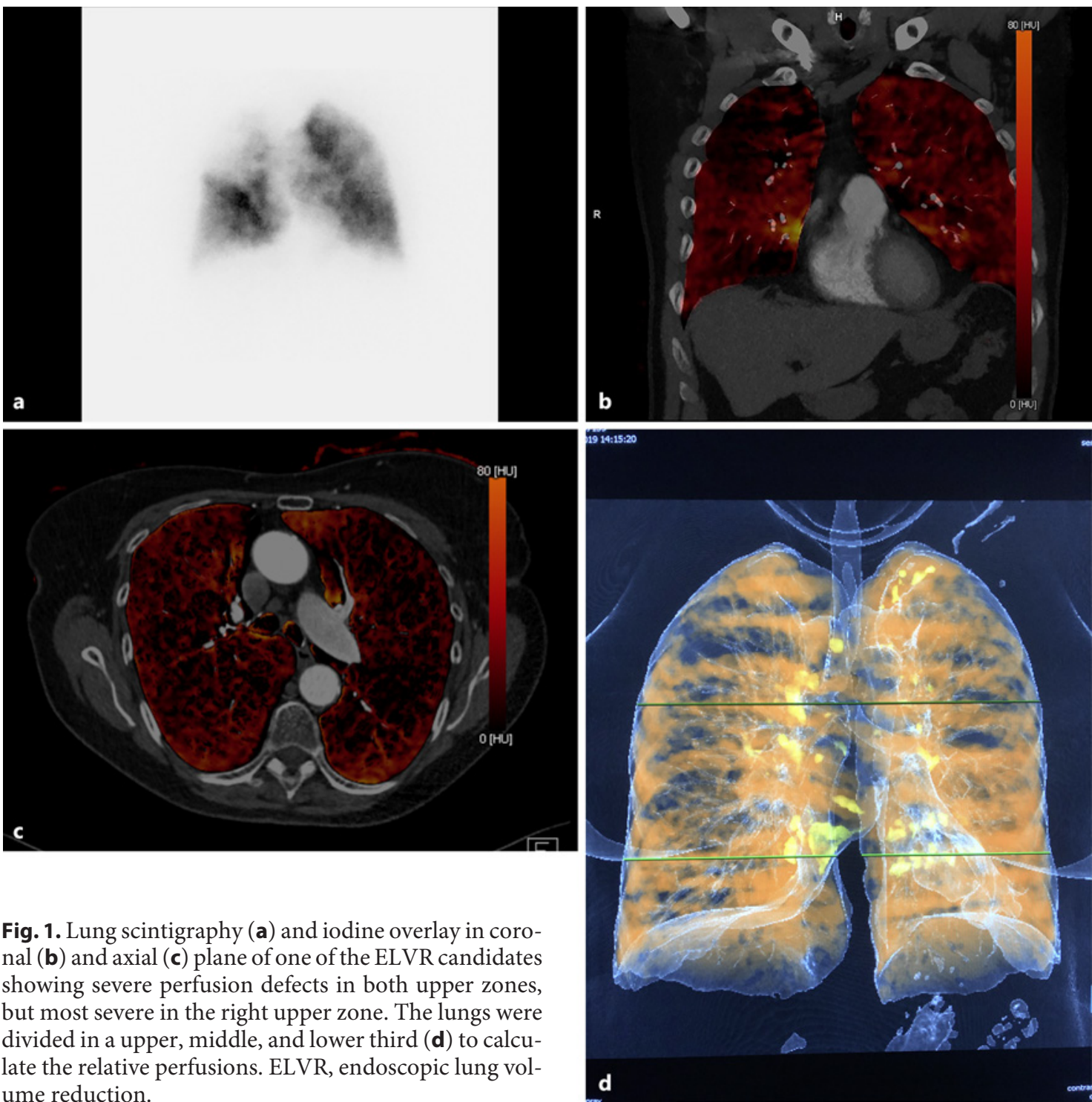

Fig. 1. Lung scintigraphy (a) and iodine overlay in coronal (b) and axial (c) plane of one of the ELVR candidates showing severe perfusion defects in both upper zones, but most severe in the right upper zone. The lungs were divided in a upper, middle, and lower third (d) to calculate the relative perfusions. ELVR, endoscopic lung volume reduction.

$80 \mathrm{kVp}, 20 \mathrm{mAs}$ and reconstructed at $1 \mathrm{~mm}$ slices. The HRCT was followed by a dual-energy contrast-enhanced CT (DCE-CT). DCE-CT imaging was performed using $90 \mathrm{kVp} / 46 \mathrm{mAs}$ (dataset A) and $150 \mathrm{kVp} / 40 \mathrm{mAs}$ setting (dataset B) $10 \mathrm{~s}$ after injection of $50 \mathrm{~mL}$ i.v. contrast at a flow rate of $5 \mathrm{~mL} / \mathrm{s}$ and included the whole lungs in a caudal-cranial direction. The 10s delay was chosen based on previous work by Das et al. (unpublished data) and is 2 s longer than the delay that is used for pulmonary emboli detection to ensure that the contrast is in the pulmonary parenchyma at time of scanning. Patients were advised to do a breath-hold at tidal volume to avoid increased perfusion in the lower lobes due to inspiration and to be able to compare the images to the perfusion scintigraphy results.

Images were sent to a Syngo.via workstation (Siemens Healthcare, Forchheim, Germany), which contains postprocessing software, and analyzed using the dual-energy mode. A virtual noncontrast-enhanced CT and an iodine map were calculated with this software (Fig 1b, c). Pulmonary perfusion was estimated by assessing the increase in density in Hounsfield units for each area of interest. The regional distribution of pulmonary perfusion at $99 \mathrm{mTc}$ perfusion scintigraphy was assessed for both lungs and for the upper, middle, and lower thirds of the lungs and presented as percentage of the total perfusion. For the current analyses, the same regions of interest were chosen for the CT images and relative contributions were calculated as percentage of the total increase in density (Fig. 1d). For the upper, middle, and lower part of each lung, the volume and increase in CT-values between the virtual non-contrast CT and the iodine map was calculated by the software. This information was used to calculated the relative contributions of each region to the total perfusion. Difference in calculated relative perfusion of each lung and of each region of interest between both techniques was calculated and plotted against the mean calculated perfusion using the method described by Bland and Altman [13]. 
Fig. 2. Relative perfusion of right (a) and left (b) lung assessed with $99 \mathrm{mTc}$ pulmonary scintigraphy ( $x$-axis) and computed from the iodine overlay calculated from contrast-enhanced dual-energy computed tomography ( $y$-axis).
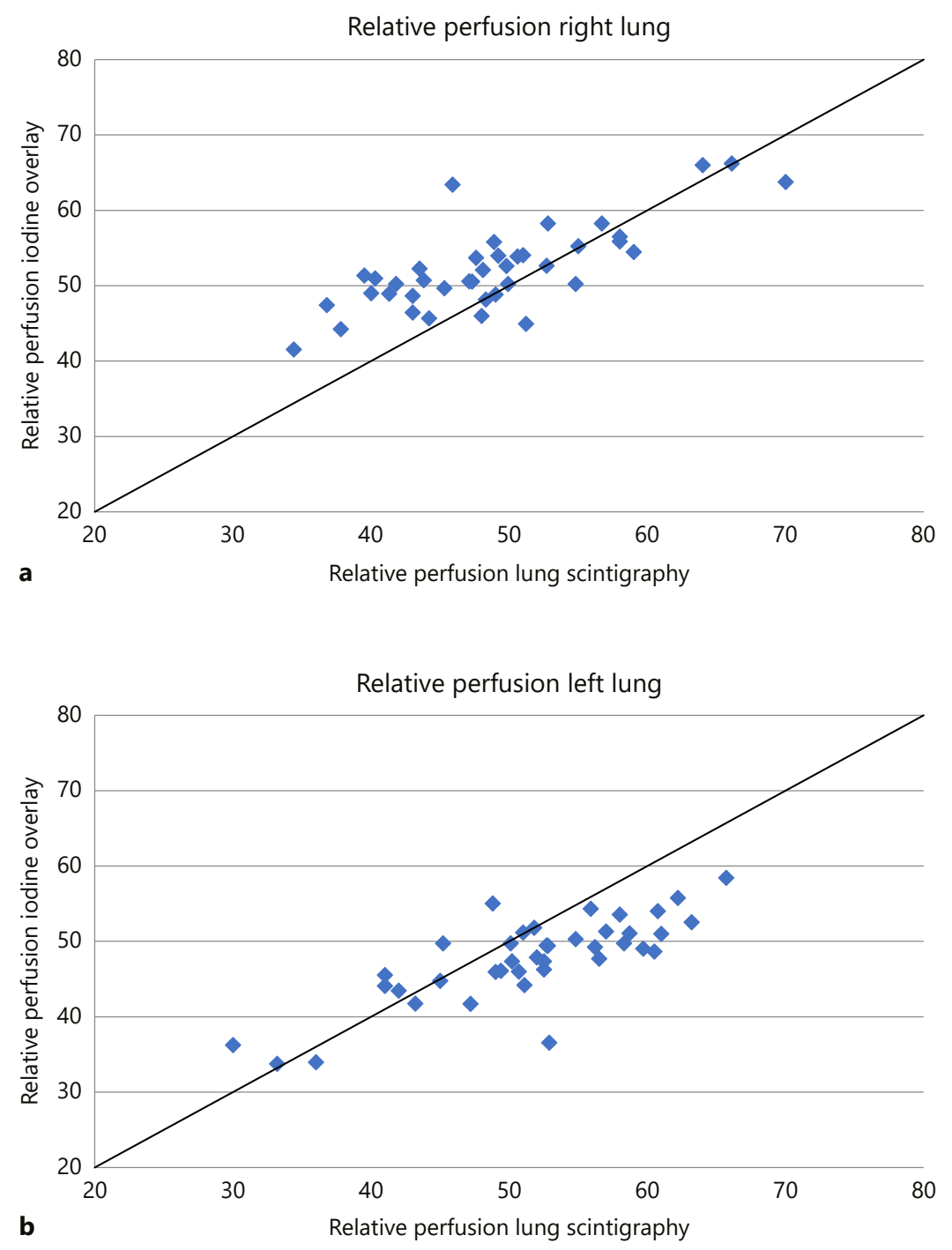

b

\section{Results}

Between February 2018 and April 2019, 42 ELVR candidates underwent both an HRCT in combination with a dual-energy contrast-enhanced CT for perfusion imaging and a Tc99 m-perfusion scintigraphy. An example of both images is shown in Figure 1. Two candidates were excluded from the study because the Syngo.via software was not able to correctly segment the lungs leaving 40 ELVR candidates available for the current analysis. Baseline demographics are presented in Table 1.

Mean age was 63.6 years and 22 candidates were female (55\%). Mean time between both examinations was $7 \pm 33$ days. Twenty-seven patients had their inspira- tory CT scans analyzed for extent of lobar destruction and for fissure integrity. In these cases, the mean destruction scores (arbitrarily defined as percentage voxels with a density $<-910 \mathrm{HU}$ ) were $52 \%, 44 \%, 45 \%, 48 \%$, and $42 \%$ for the right upper lobe, middle lobe, right lower lobe, left upper lobe, and left lower lobe, respectively. All patients had severe centrilobular emphysema with or without a limited paraseptal component, and mainly with an upper lobe predominance. In twelve cases ELVR was performed. Integrity of the fissure adjacent to the target lobe in these cases was on average 98\% (94-100\%).

Perfusion distribution among both lungs was close to a 50/50 distribution with a slightly greater perfusion of 
Fig. 3. Box and whisker plot of differences in regional perfusion. $Y$-axis shows difference between relative perfusion measured by $99 \mathrm{mTc}$ perfusion scintigraphy and relative perfusion calculated by the iodine overlay. RU, right upper; RM, right middle; RL, Right lower; LU, left upper, LM, left middle; LL, left lower.

Table 1. Baseline demographics study population

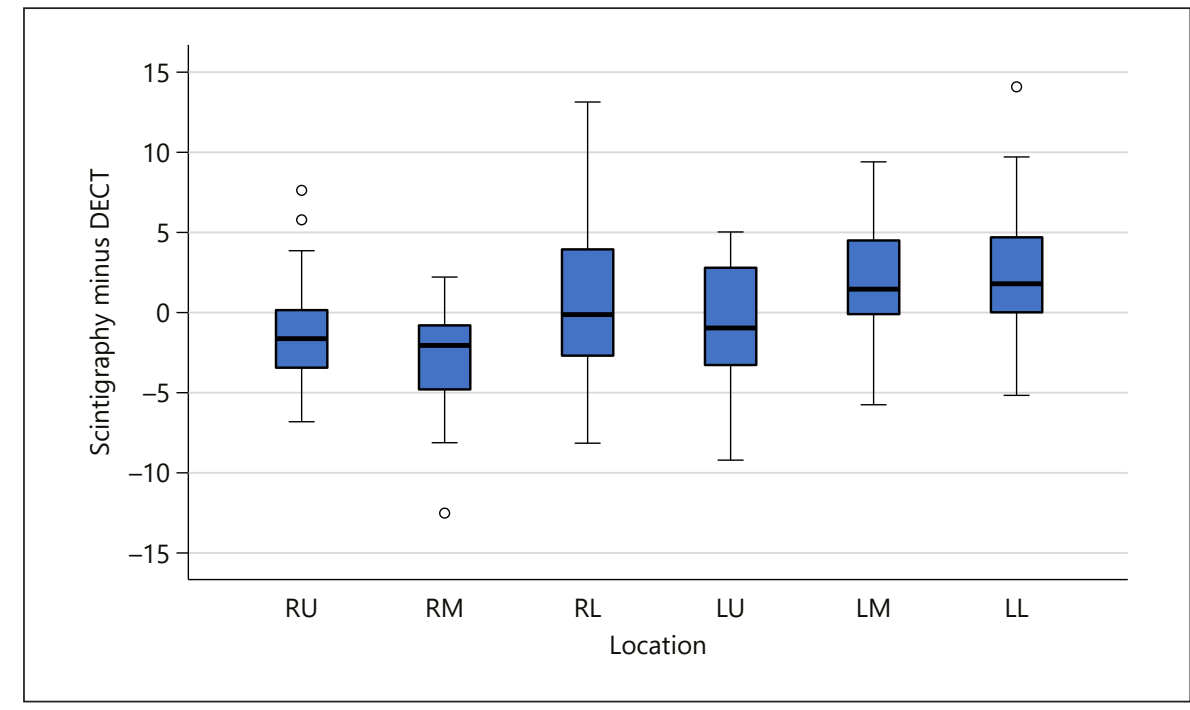

\begin{tabular}{lcl}
\hline & Mean & Range \\
\hline Age & 63.6 years & $46-75$ years \\
Male/female & $18 / 22$ & $0.4-2.9 \mathrm{~L}(16-95 \%)$ \\
FEV1, \%pred & $0.8 \mathrm{~L}(31.5)$ & $1.2-6.7 \mathrm{~L}(43-105 \%)$ \\
FVC, \%pred & $2.8 \mathrm{~L}(77.2)$ & $0.20-0.79(23-68 \%)$ \\
FEV1/FVC, \%pred & $0.3(41 \%)$ & $3.3-7.2 \mathrm{~L}(95-374 \%)$ \\
RV, \%pred & $4.8 \mathrm{~L}(226 \%)$ & $5.1-11 \mathrm{~L}(96-279 \%)$ \\
TLC, \%pred & $7.6 \mathrm{~L}(139 \%)$ & $130-607 \mathrm{~m}(20-95 \%)$ \\
6MWT, \%pred & $357 \mathrm{~m}(58 \%)$ & $1.8-8.8 \mathrm{mmol} / \mathrm{min} / \mathrm{kPa}(21-77 \%)$ \\
Dco, \%pred & $3.6 \mathrm{mmol} / \mathrm{min} / \mathrm{kPa}(41.5 \%)$ & \\
\hline
\end{tabular}

TLC, total lung capacity. the left lung assessed with perfusion scintigraphy and a slightly greater perfusion of the right lung assessed by DCE-CT (both $p<0.001$ ). The 2 techniques showed a good correlation with $r=0.8$ for both right and left lung. Mean difference was 3.5\% $( \pm 5.0 \%)$ as shown in Figure 2. Differences in calculated relative perfusion of the right lung were mainly due to differences in calculated perfusion in the upper and middle part of the right lung, while differences in relative perfusion of the left lung were mainly due to a greater relative perfusion of lower part of the left lung at $99 \mathrm{mTc}$ perfusion scintigraphy. We did not see a bias toward a greater perfusion in the lower lobes at $\mathrm{CT}$, indicating that patients followed the breathing instructions well. No correlation was seen between difference in calculated perfusion distribution and time between the 2 examinations ( $p=0.18$ ).

The relative perfusion was slightly lower for the upper thirds and greatest for the middle thirds, which had also the largest volumes due to the shape of the lungs. Differences in calculated perfusion were calculated for each of the 6 regions of interest and the differences per region are shown in Figure 3. Mean differences for each region of interest were close to zero and interquartile ranges were all between $-5 \%$ and $5 \%$.

In Figure 4, the Bland and Altman plots of the 6 regions of interest are displayed. The relative perfusion of the upper lobes was lowest as expected in a population with severe smoking-related advanced destructive emphysema. The mean difference in measured pulmonary perfusion between the 2 techniques ranged from $-2.8 \%$ to $2.3 \%$. The lower limit of agreement ranged from $-8.9 \%$ to $-4.6 \%$ and upper limit ranged from $3.3 \%$ to $10.0 \%$.

Three patients treated with ELVR had a homogeneous distribution of emphysema. In these cases, the perfusion distribution had an important role in the selection of the 


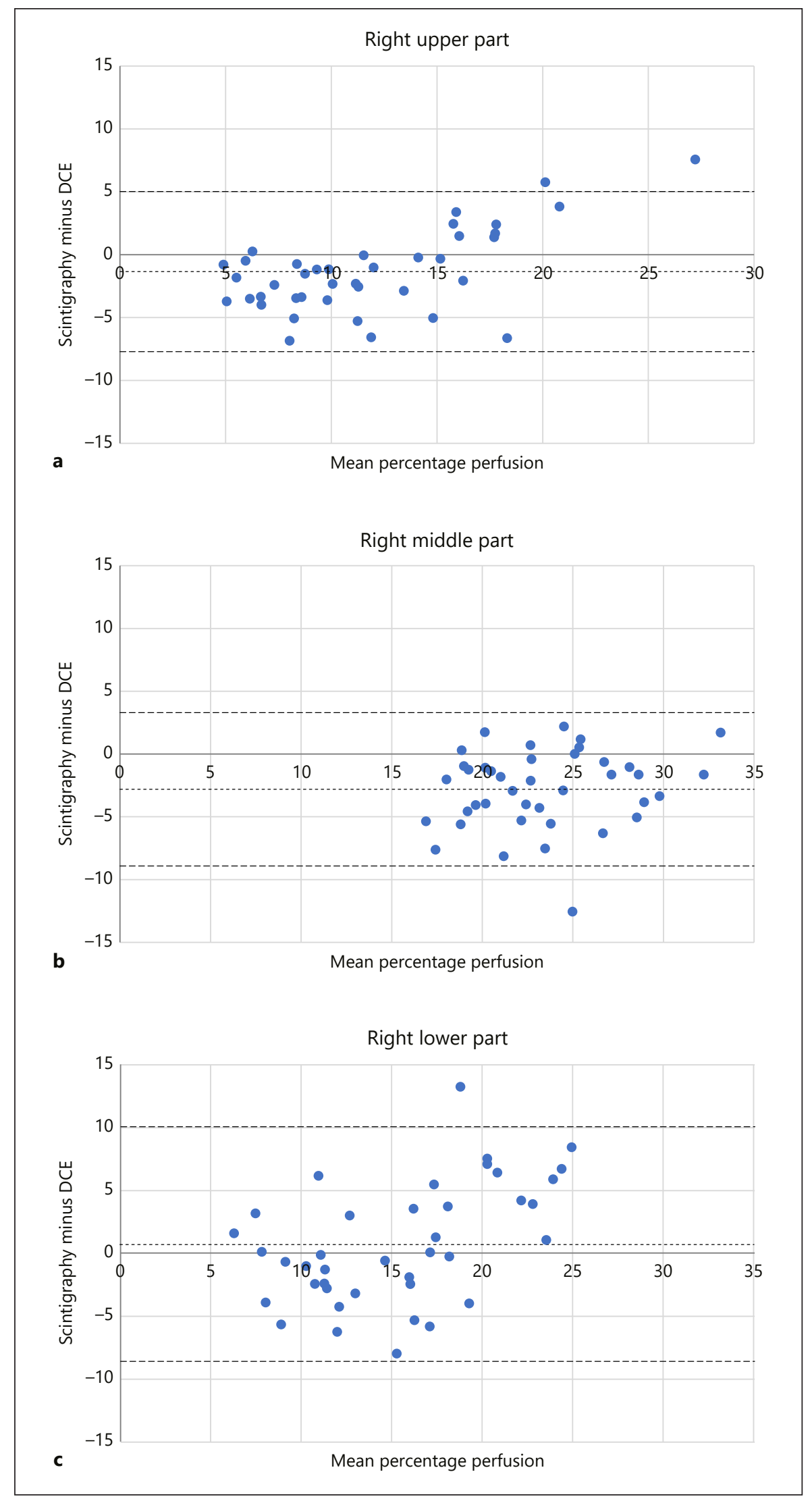

Fig. 4. Bland and Altman plots of the 6 regions of interest.

(Figure continued on next page.) 


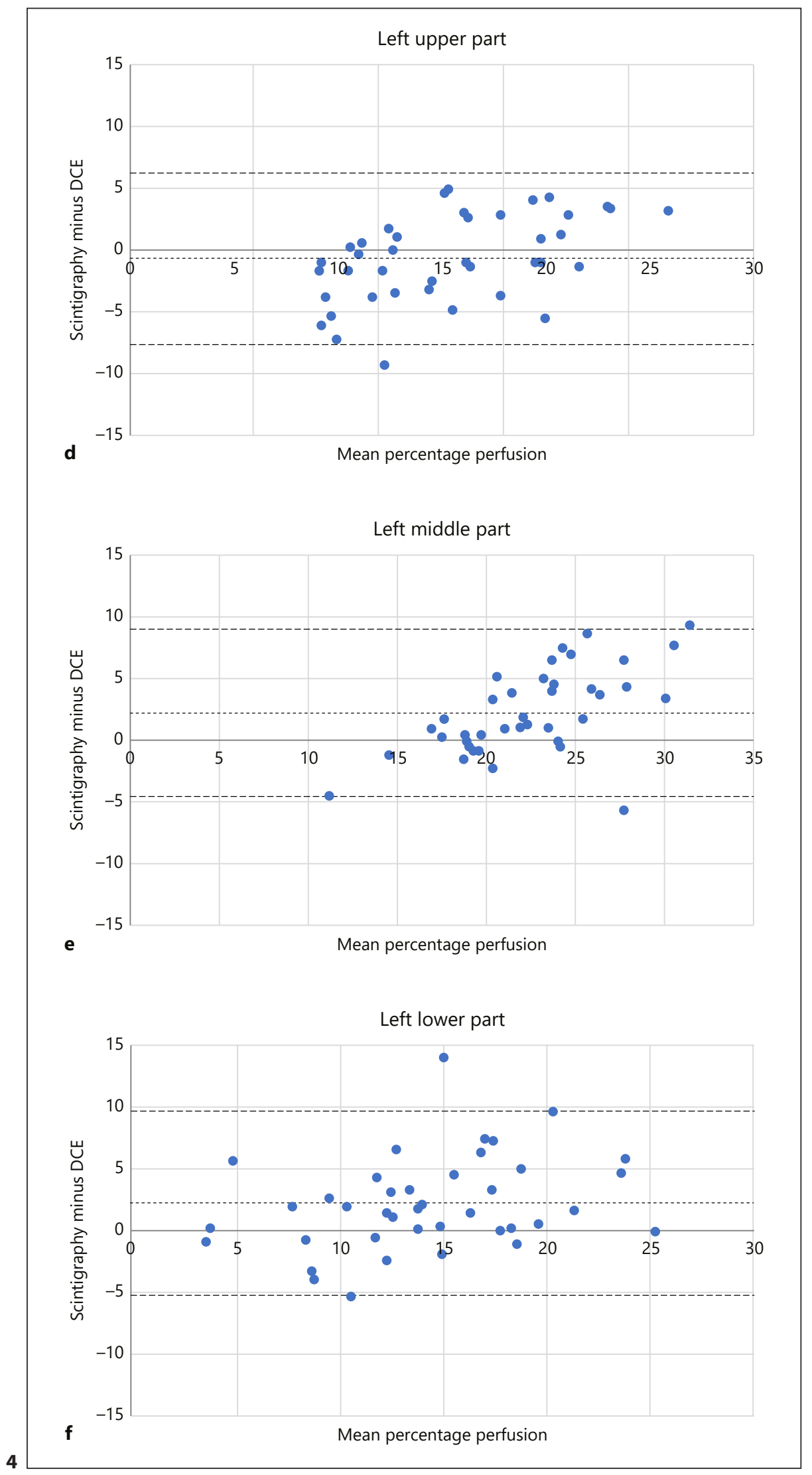


target lobe. The selected target lobe was independent of the technique of perfusion assessment in all cases. In the 9 treated cases with heterogeneous emphysema distribution, the perfusion distribution was less important, but was taken into consideration. In none of the cases the technique of perfusion assessment had impact on target lobe selection.

\section{Discussion}

In this analysis, we showed that pulmonary perfusion assessment using the iodine overlay calculated from the post-contrast dual-energy images is comparable to pulmonary perfusion quantification by lung perfusion scintigraphy, even though the first technique is a single shot technique, while the latter assesses perfusion during a longer period. The assessment of lung perfusion distribution prior to ELVR recommended, but not mandatory [8]. However, low perfusion of the upper lobes, as assessed by ventilation/perfusion scans was shown to associate with less mortality in patients treated with lung volume reduction surgery [14]. Argula et al. [15] analyzed the impact of target lobe perfusion as dichotomous parameter and showed that treated patients in the group with low perfusion in the target lobe had a greater increase in the $6 \mathrm{MWT}$ than treated patients in the group with high perfusion in the target lobe. Thomson et al. [16] showed that perfusion of the target lobe at baseline was not predictive of outcome, but patients with high perfusion of the ipsilateral nontarget lobe demonstrated greater improvements in 6MWT.

Dual-energy computed tomography calculates an iodine map by acquiring images with different energies. Although this is a non-dynamic technique, it has been shown that perfusion distribution estimated from the iodine overlay is highly comparable to the results provided by $99 \mathrm{~m}$ Technetium scintigraphy $[10,11,17]$. So far, pulmonary iodine maps have mainly been used to detect perfusion defects due to pulmonary emboli. And although a previous study showed good to very good agreement between these 2 imaging techniques [17], for detection of perfusion defects due to pulmonary emboli only a qualitative assessment of the defects is done (present/ absent), while in the work-up for ELVR quantification is required.

In our study, we evaluated the application of the calculated iodine overlay in assessment of perfusion distribution over both lungs in over the upper, middle, and lower parts of the lungs in the work-up of ELVR candi-

CT versus Scintigraphy to Evaluate Lung

Perfusion in Emphysema dates, who currently undergo perfusion scintigraphy for this assessment. We showed that the results of both techniques correlate well, but also major outliers are seen with differences in relative regional perfusion up to $10 \%$ of total perfusion; however, this did not result in changes in clinical decision-making. Imaging is used to define a target lobe and with the current techniques, perfusion distribution is not assessed by lobe. With the planar technique no anatomical information is delivered, which is required for lobar quantification. Therefore, in all patients the perfusion scintigraphy was combined with a Single Photon Emission Computed Tomography (SPECT) for anatomical information. Using the SPECT imaging a visual estimation could be done which lobe was better perfusion since for example, in the middle third of both lung perfusion of the lower part of the upper lobe, on the right side the middle lobe and the upper part of the lower lobe is measured. DCE-CT combined with HRCT would save the patient an additional examination since the HRCT is mandatory is patient selection and to define a potential target lobe. While planar perfusion scintigraphy lacks anatomical information required for lobe selection, iodine overlay images performed with CT-scanning can provide the exact location of the perfusion at time of scanning, showing the perfusion defects in the destroyed areas.

For $99 \mathrm{mTc}$ perfusion scintigraphy, patients were asked to breath normally at tidal volume. In order to exclude increased perfusion in the lower lobes due to deep inspiration, patients were asked to hold their breath at tidal volume at acquiring the dual-energy CT scans. We did not determine a systematic difference between the 2 techniques toward a greater perfusion in the lower regions at the iodine maps, suggesting that the patients followed the instructions.

A possible explanation of higher calculated "perfusion" of the right lobe at the iodine overlays could be the artifacts due to beam hardening with an area of greater increase in Hounsfield units next to the upper vena cava, which is also noted in Figure 2. However, this artifact was also seen in subjects who did not show a relevant difference between both techniques (defined as differences close to zero). We also noted beam hardening artifacts next to the subclavian vein, but no relationship between difference in pulmonary perfusion between both techniques and i.v. contrast injection side was seen. In the majority of patients, i.v. contrast was injected in the left antecubital vein.

In an early dual-energy CT study in smokers, Pansini et al. [12] already showed that subjects with severe em- 
physema have a lower attenuation enhancement than smokers without emphysema and non-smokers. Provost et al. [18] showed excellent inter- and intraobserver agreement for perfusion quantification with $99 \mathrm{mTc}$ perfusion SPECT/CT, with measurement variation up to only $2 \%$ in $96 \%$ of the cases. This is much lower than the differences we noted in the current study. However, this study was performed on a single examination, excluding variation due to changes in lung shape and volume.

The major limitation of the planar technique is the inherent combined result of different lobes in the middle thirds. In these cases, visual assessment of the SPECT-CT images and especially the iodine overlays is helpful and used in decision-making.

With new software packages, it is possible to assess lobar perfusion at lung scintigraphy data. However, these software packages are not yet widespread. Future research will focus on lobar perfusion distribution both at lung scintigraphy and iodine overlay data.

A major limitation of this study is the lack of a gold standard. As far as our knowledge is concerned, for neither of the techniques, the inter-examination variability is known, so we were not able to relate the reported differences in measured perfusion between both techniques to normal measurement variation for lung perfusion scintigraphy, which is currently the standard of care and recommended by the expert panel for ELVR [8].

Another limitation is that the Syngo.via software provides increase in density between the virtual non-contrast-enhanced CT scan and the iodine map, but the relative contributions of each area of interest to the total perfusion needed to be calculated manually. Although visual judgment of the iodine maps resulted in a good estimation of the relative contribution of each region of interest, calculation of the percentage of residual perfusion in each region of interest was a time-consuming task and cannot easily be applied in clinical practice, yet. However, a newer version of Syngo.via being able to calculate this automatically, is being developed. Our results show that, when an automated quantification tool for the perfusion distribution, the perfusion scintigraphy can be omitted.

In conclusion, we showed that pulmonary perfusion quantification with dual-energy contrast-enhanced CT is comparable to the results from $99 \mathrm{mTc}$ perfusion scintigraphy, but also major differences in quantified lung perfusion distribution are seen. However, this had no impact on clinical decision-making in potential ELVR candidates.

\section{Statement of Ethics}

The presented research was conducted according to the Declaration of Helsinki. The study was approved by the local institutional review board and a waiver was received due to its retrospective nature. The authors have no ethical conflicts to disclose.

\section{Conflict of Interest Statement}

The authors have no conflicts of interest to disclose.

\section{Funding Sources}

R.P., D.J.S., and L.V. received a grant from the Dutch Lung Foundation for the SOLVE trial (Grant No. 5.1.17.171).

\section{Author Contributions}

H.G.: substantial contribution to the conception and design of the work, interpretation, and analysis of the data; drafting and revising the manuscript and final approval; and accountable for accuracy and integrity of the work. K.W.: substantial contribution to the conception and design of the work, interpretation, and analysis of the data; drafting and revising the manuscript and final approval; and accountable for accuracy and integrity of the work. R.P.: substantial contribution to the conception and design of the work; revising the manuscript and final approval; and accountable for accuracy and integrity of the work. C.M.: substantial contribution to the conception and design of the work; revising the manuscript and final approval; and accountable for accuracy and integrity of the work. D.J.S.: substantial contribution to the conception and design of the work; revising the manuscript and final approval; and accountable for accuracy and integrity of the work. L.V.: substantial contribution to the conception and design of the work, interpretation, and analysis of the data; drafting and revising the manuscript and final approval; and accountable for accuracy and integrity of the work.

\section{References}

1 Gompelmann D, Heinhold T, Rötting M, Bischoff E, Kontogianni K, Eberhardt R, et al. Long-term follow up after endoscopic valve therapy in patients with severe emphysema. Ther Adv Respir Dis. 2019;13: 1753466619866101.

2 Hartman JE, Vanfleteren LEGW, van Rikxoort EM, Klooster K, Slebos DJ. Endobronchial valves for severe emphysema. Eur Respir Rev. 2019;28(152):180121

3 Herth FJF, Slebos DJ, Criner GJ, Valipour A, Sciurba F, Shah PL. Endoscopic lung volume reduction: an expert panel recommendationUpdate 2019. Respiration. 2019;97(6):54857. 
4 Valipour A, Slebos DJ, Herth F, Darwiche K, Wagner M, Ficker JH, et al. Endobronchial valve therapy in patients with homogeneous emphysema. Results from the IMPACT study. Am J Respir Crit Care Med. 2016; 194(9):1073-82.

5 Koster TD, van Rikxoort EM, Huebner RH, Doellinger F, Klooster K, Charbonnier JP, et al. Predicting lung volume reduction after endobronchial valve therapy is maximized using a combination of diagnostic tools. Respiration. 2016;92(3):150-7.

6 van Geffen WH, Klooster K, Hartman JE, Ten Hacken NHT, Kerstjens HAM, Wolf RFE, et al. Pleural adhesion assessment as a predictor for pneumothorax after endobronchial valve treatment. Respiration. 2017;94(2):224-31.

7 Slebos DJ, Shah PL, Herth FJ, Valipour A. Endobronchial valves for endoscopic lung volume reduction: best practice recommendations from expert panel on endoscopic lung volume reduction. Respiration. 2017;93(2):138-50.

8 Herth FJF, Slebos DJ, Criner GJ, Shah PL. Endoscopic lung volume reduction: an expert panel recommendationUpdate 2017. Respiration. 2017;94(4):380-8.
9 Pontana F, et al. Lung perfusion with dualenergy multidetector-row CT (MDCT): feasibility for the evaluation of acute pulmonary embolism in 117 consecutive patients. Acad Radiol. 2008;15(12):1494-504.

10 Masy M, Giordano J, Petyt G, HosseinFoucher C, Duhamel A, Kyheng M, et al. Dual-energy CT (DECT) lung perfusion in pulmonary hypertension: concordance rate with V/Q scintigraphy in diagnosing chronic thromboembolic pulmonary hypertension (CTEPH). Eur Radiol. 2018;28(12):5100-10.

11 Renapurkar RD, Bolen MA, Shrikanthan S, Bullen J, Karim W, Primak A, et al. Comparative assessment of qualitative and quantitative perfusion with dual-energy CT and planar and SPECT-CT V/Q scanning in patients with chronic thromboembolic pulmonary hypertension. Cardiovasc Diagn Ther. 2018; 8(4):414-22.

12 Pansini V, Remy-Jardin M, Faivre JB, Schmidt B, Dejardin-Bothelo A, Perez T, et al. Assessment of lobar perfusion in smokers according to the presence and severity of emphysema: preliminary experience with dual-energy CT angiography. Eur Radiol. 2009;19(12):2834.

13 Bland JM, Altman D. Statistical methods for assessing agreement between two methods of clinical measurement. Lancet. 1986; 327(8476):307-10
14 Chandra D, Lipson DA, Hoffman EA, Hansen-Flaschen J, Sciurba FC, Decamp MM, et al. Perfusion scintigraphy and patient selection for lung volume reduction surgery. Am J Respir Crit Care Med. 2010;182(7):937-46.

15 Argula RG, Strange C, Ramakrishnan V, Goldin J. Baseline regional perfusion impacts exercise response to endobronchial valve therapy in advanced pulmonary emphysema. Chest. 2013;144(5):1578-86.

16 Thomsen C, Theilig D, Herzog D, Poellinger A, Doellinger F, Schreiter N, et al. Lung perfusion and emphysema distribution affect the outcome of endobronchial valve therapy. Int J Chron Obstruct Pulmon Dis. 2016;11: 1245-59.

17 Thieme SF, Becker CR, Hacker M, Nikolaou $\mathrm{K}$, Reiser MF, Johnson TR. Dual energy CT for the assessment of lung perfusioncorrelation to scintigraphy. Eur J Radiol. 2008;68(3): 369-74.

18 Provost K, Leblond A, Gauthier-Lemire A, Filion É, Bahig H, Lord M. Reproducibility of lobar perfusion and ventilation quantification using SPECT/CT segmentation software in lung cancer patients. J Nucl Med Technol. 2017;45(3):185-92. 